Irish Math. Soc. Bulletin

Number 80, Winter 2017, 35-44

ISSN 0791-5578

\title{
Modular Metric Spaces
}

\author{
HANA ABOBAKER AND RAYMOND A. RYAN
}

\begin{abstract}
We give a short introduction to the theory of modular metric spaces, including some fixed point theorems due to Chistyakov. We give new proofs of these theorems, inspired by Palais's approach to the Banach fixed point theorem
\end{abstract}

\section{INTRODUCTION}

We begin with a simple motivating example. Let $X$ be the set of all points above water on the earth's surface. For two points $x, y$, let us denote by $v_{t}(x, y)$ the average speed required to travel directly over land from $x$ to $y$ in a time $t$. What are the properties of the function $v_{t}(x, y)$ ?

Clearly, if we fix $x$ and $y$, then $v_{t}(x, y)$ takes nonnegative values and is a non-increasing function of $t$. And of course, this function is symmetric in $x$ and $y$. But there is an issue we have glossed over what if $x$ and $y$ lie in different landmasses? Since we are required to travel by land, it is impossible to get from $x$ to $y$ in a time $t$, no matter how fast we travel. As we would like our speed function to be defined in all circumstances, it is reasonable to allow extended real values and to assign the value $v_{t}(x, y)=\infty$ in this case.

To summarize, we now have a function taking $t>0$ and $x, y \in X$ to $v_{t}(x, y) \in[0, \infty]$ that is symmetric in $x, y$ and non-increasing in $t$. There is one further property worthy of note. A simple calculation with average speeds shows that

$$
v_{s+t}(x, y) \leq v_{s}(x, z)+v_{t}(z, y)
$$

for all $s, t>0$ and all $x, y, z \in X$.

2010 Mathematics Subject Classification. Primary 54H25; Secondary 55M20, $58 \mathrm{C} 30$

Key words and phrases. modular metric, fixed point.

Received on 14-11-2016; revised 29-6-2017.

The work of the first author was supported by a grant from the Ministry of Education of Libya, to whom she expresses her gratitude.

(C)2017 Irish Mathematical Society 
In 2006, Vyacheslav Chistyakov [4, 2] introduced the concept of a metric modular on a set, inspired partly by the classical linear modulars on function spaces employed by Nakano and other in the 1950s. Our average speed function is an example of a modular in the sense of Chistyakov.

The concept of a modular on a vector space was introduced by Nakano in 1950 [10] and refined by Musielak and Orlicz in 1959 [9]. In the formulation given by Kowzslowski [7, 6], a modular on a vector space $X$ is a function $m: X \rightarrow[0, \infty]$ satisfying

(1) $m(x)=0$ if and only if $x=0$;

(2) $m(a x)=m(x)$ for every $a \in \mathbb{R}$ with $|a|=1$;

(3) $m(a x+b y) \leq m(x)+m(y)$ if $a, b \geq 0$ and $a+b=1$.

A modular $m$ is said to be convex if, instead of (3), it satisfies the stronger property

(3') $m(a x+b y) \leq a m(x)+b m(y)$ if $a, b \geq 0$ and $a+b=1$.

Given a modular $m$ on $X$, the modular space is defined by

$$
X_{m}=\{x \in X: m(a x) \rightarrow 0 \text { as } a \rightarrow 0\}
$$

It is possible to define a corresponding $\mathrm{F}$-norm (or a norm when $m$ is convex) on the modular space. The Orlicz spaces $L^{\varphi}$ are examples of this construction [12].

The notion of a metric modular on an arbitrary set was introduced in 2006 by Chistyakov as a generalization of these ideas. Chistyakov's formulation provides a good framework in which to study fixed point phenomena.

\section{Modular Metric SPACES}

We start with the definition given by Chistyakov.

Definition 1. Let $X$ be a nonempty set. A metric modular on $X$ is a function

$$
w:(0, \infty) \times X \times X \rightarrow[0, \infty],
$$

written as $(\lambda, x, y) \mapsto w_{\lambda}(x, y)$, that satisfies the following axioms:

(1) $w_{\lambda}(x, y)=0$ if and only if $x=y$ for all $\lambda>0$ and $x, y \in X$

(2) $w_{\lambda}(x, y)=w_{\lambda}(y, x)$ for all $\lambda>0$ and $x, y \in X$

(3) $w_{\lambda+\mu}(x, y) \leq w_{\lambda}(x, z)+w_{\mu}(y, z)$ for all $\lambda, \mu>0$ and $x, y, z \in$ $X$. 
If the context is clear, we refer to a metric modular simply as a modular. A modular $w$ is said to be strict if it has the following property: given $x, y \in X$ with $x \neq y$, we have $w_{\lambda}(x, y)>0$ for all $\lambda>0$.

A modular $w$ on $X$ is said to be convex if, instead if (3), it satisfies the stronger inequality

$$
w_{\lambda+\mu}(x, y) \leq \frac{\lambda}{\lambda+\mu} w_{\lambda}(x, z)+\frac{\mu}{\mu+\lambda} w_{\mu}(z, y),
$$

for all $\lambda, \mu>0$ and $x, y, z \in X$.

Let $(X, d)$ be a metric space with at least two points. There are several ways to define a metric modular on $X$.

\section{Example 2.1.}

$$
w_{\lambda}(x, y)=d(x, y)
$$

In this case, property (3) in the definition of a modular is just the triangle inequality for the metric. This modular is not convex - just take $z=y$ and $\mu=\lambda$ in (1).

\section{Example 2.2.}

$$
w_{\lambda}(x, y)=\frac{d(x, y)}{\lambda}
$$

In this case, we can think of $w_{\lambda}(x, y)$ as the average velocity required to travel from $x$ to $y$ in time $\lambda$. A simple calculation with the triangle inequality shows that this modular is convex.

\section{Example 2.3.}

$$
w_{\lambda}(x, y)=\left\{\begin{array}{l}
\infty \quad \text { if } \lambda<d(x, y), \\
0 \quad \text { if } \lambda \geq d(x, y)
\end{array}\right.
$$

This simple example could be seen as an extreme case of the velocity metaphor - if the time available is less than $d(x, y)$, then it is impossible to travel from $x$ to $y$, but if the time is at least $d(x, y)$ then we can travel instantaneously. This modular is also convex.

We now look at some of the basic properties of modulars.

Proposition 2.4. Let $w$ be a modular on the set $X$.

(a) For every $x, y \in X$, the function $\lambda \mapsto w_{\lambda}(x, y)$ is non-increasing. 
(b) Let $w$ be a convex modular. For $x, y \in X$, if $w_{\lambda}(x, y)$ is finite for at least one value of $\lambda$, then $w_{\lambda}(x, y) \rightarrow 0$ as $\lambda \rightarrow \infty$ and $w_{\lambda}(x, y) \rightarrow \infty$ as $\lambda \rightarrow 0+$.

(c) If $w$ is a convex modular, then the function $v_{\lambda}(x, y)=\frac{w_{\lambda}(x, y)}{\lambda}$ is a modular on $X$.

Proof.

(a) follows from property (3) of modulars, taking $z=y$.

(b) Taking $z=y$ in equation (1) gives

$$
w_{\lambda^{\prime}}(x, y) \leq \frac{\lambda}{\lambda^{\prime}} w_{\lambda}(x, y)
$$

whenever $\lambda^{\prime}>\lambda$. Choosing $\lambda$ for which $w_{\lambda}(x, y)$ is finite, we see that $w_{\lambda^{\prime}}(x, y) \rightarrow 0$ as $\lambda^{\prime} \rightarrow \infty$. And if we fix $\lambda^{\prime}$ such that $w_{\lambda^{\prime}}(x, y)<\infty$, we get $w_{\lambda}(x, y) \rightarrow \infty$ as $\lambda \rightarrow 0+$.

(c) It is obvious that $v$ satisfies the first two properties of a modular Property (3) for $v$ follows easily from the convexity condition on $w_{\lambda}$.

\section{Modular SETS AND MOdular CONVERGENCE}

Given a modular $w$ on $X$ and a point $x_{0}$ in $X$, the two sets

$$
X_{w}\left(x_{0}\right)=\left\{x \in X: w_{\lambda}\left(x, x_{0}\right) \rightarrow 0 \text { as } \lambda \rightarrow \infty\right\}
$$

and

$$
X_{w}^{*}\left(x_{0}\right)=\left\{x \in X: \exists \lambda>0 \text { such that } w_{\lambda}\left(x, x_{0}\right)<\infty\right\}
$$

are each known as Modular Sets around $x_{0}$. These sets can be thought of as comprising all the points that are "accessible" in some sense from $x_{0}$. In both cases, the modular sets form a partition of $X$. In our motivating example, the modular sets are the individual land masses.

It is clear that $X_{w}\left(x_{0}\right) \subset X_{w}^{*}\left(x_{0}\right)$ in general and Example 1 shows that this inclusion may be strict. While there is some ambiguity in using the same term for these two types of sets, in the sequel we shall only be working with modular sets of the form $X_{w}^{*}\left(x_{0}\right)$.

Proposition 3.1. If $w$ is convex then

$$
X_{w}\left(x_{0}\right)=X_{w}^{*}\left(x_{0}\right) .
$$

Proof. This follows immediately from Proposition 2.4 (b).

We now turn our attention to some notions of convergence. 
Definition 2. Let $w$ be a modular on $X$. A sequence $\left(x_{n}\right)$ in $X$ is said to be $w$-convergent (or modular convergent) to an element $x \in X$ if there exists a number $\lambda>0$, possibly depending on $\left(x_{n}\right)$ and $x$, such that $\lim _{n \rightarrow \infty} w_{\lambda}\left(x_{n}, x\right)=0$ A sequence $\left(x_{n}\right)$ in $X$ is said to be $w$-Cauchy if there exists $\lambda>0$, possibly depending on the sequence, such that $w_{\lambda}\left(x_{m}, x_{n}\right) \rightarrow 0$ as $m, n \rightarrow \infty$. $X$ is said to be $w$-complete if every $w$-Cauchy sequence is $w$-convergent.

\section{Metrics on the modular set}

Let $w$ be a modular on $X$ and let $X_{w}$ be any one of the modular sets defined by $w$. Then the formula

$$
d_{w}(x, y)=\inf \left\{\lambda>0: w_{\lambda}(x, y) \geq \lambda\right\}, \quad \forall x, y \in X_{w}
$$

defines a metric on $X_{w}[4]$.

If the modular $w$ is convex, then the modular space can be endowed with another a metric $d_{w}^{*}$ given by

$$
d_{w}^{*}(x, y)=\inf \left\{\lambda>0: w_{\lambda}(x, y) \leq 1\right\} .
$$

These metrics on the modular set are strongly equivalent:

$$
d_{w} \leq d_{w}^{*} \leq 2 d_{w}
$$

We refer to [4] for details.

The following result shows the relationship between modular and metric convergence. The proof can be found in [4].

Proposition 3.2. Let $w$ be a convex modular on X. Given a sequence $x_{n}$ for $X_{w}^{*}$ and an element $x \in X_{w}^{*}$, we have:

$$
\lim _{n \rightarrow \infty} d_{w}^{*}\left(x_{n}, x\right)=0 \Longleftrightarrow \lim _{n \rightarrow \infty} w_{\lambda}\left(x_{n}, x\right)=0 \text { for every } \lambda>0 .
$$

Chistyakov gives an example to show that modular convergence is strictly weaker than metric convergence in general [3]

\section{Fixed Point Theorems}

In 2011, Chistyakov generalized the Banach fixed point theorem to the setting of modular metric spaces. Consider the definition of a contraction on a metric space: $d(T x, T y) \leq k d(x, y)$ for all $x, y$, where $k$ is some constant between 0 and 1. Looking at Examples 1 and 2 , we see that there are at least two ways to generalise this to modular metric spaces. Chistyakov gives two definitions: 
Definition 3. Let $w$ be a modular on a set $X$ and let $X_{w}^{*}$ be a modular set. A mapping $T: X_{w}^{*} \rightarrow X_{w}^{*}$ is said to be modular contractive (or a $w$-contraction) if there exist numbers $k \in(0,1)$ and $\lambda_{0}>0$ such that

$$
w_{k \lambda}(T x, T y) \leq w_{\lambda}(x, y)
$$

for all $0<\lambda \leq \lambda_{0}$ and all $x, y \in X_{w}^{*}$.

His second definition is stronger:

Definition 4. A mapping $T: X_{w}^{*} \rightarrow X_{w}^{*}$ is said to be strongly modular contractive (or a strong $w$-contraction) if there exist numbers $0<k<1$ and $\lambda_{0}>0$ such that

$$
w_{k \lambda}(T x, T y) \leq k w_{\lambda}(x, y)
$$

for all $0<\lambda \leq \lambda_{0}$ and all $x, y \in X_{w}^{*}$.

Chistyakov proved fixed point theorems for modular contractive and strongly modular contractive mappings. Rather than follow his proofs, our approach to these results is inspired by Richard Palais's proof of the Banach fixed point theorem [11].

Suppose that $T$ is a contraction on a metric space $(X, d)$ with contraction constant $k$. Thus, we have $d(T x, T y) \leq k d(x, y)$ for all $x, y \in X$. Combining this with an application of the triangle inequality to the points $x, y, T x$ and $T y$, we get the inequality

$$
d(x, y) \leq \frac{d(x, T x)+d(y, T y)}{1-k} .
$$

Palais called this the Fundamental Contraction Inequality. It is a key ingredient in his proof, where it is used to establish the Cauchy property for the sequence generated by iterating the mapping $T$ on some initial point.

We begin with a variant of Palais's inequality for modular contractive mappings.

Proposition 4.1 (Fundamental Modular Contraction Inequality). Let $w$ be a convex modular in $X$, let $T: X_{w}^{*} \rightarrow X_{w}^{*}$ be a modular contractive mapping, with $w_{k \lambda}(T x, T y) \leq w_{\lambda}(x, y)$ for $0<\lambda \leq \lambda_{0}$. Let $\lambda_{1}, \lambda_{2} \geq 0, \lambda_{1}+\lambda_{2}=(1-k) \lambda$, where $0<\lambda<\lambda_{0}$. Then

$$
w_{\lambda}(x, y) \leq \frac{\lambda_{1} w_{\lambda_{1}}(x, T x)+\lambda_{2} w_{\lambda_{2}}(y, T y)}{\lambda(1-k)}
$$

for every $x, y \in X_{w}^{*}$ 
Proof. By the convex property, taking $\lambda=\lambda_{1}+k \lambda+\lambda_{2}$, we get

$$
w_{\lambda_{1}+k \lambda+\lambda_{2}}(x, y) \leq \frac{\lambda_{1}}{\lambda} w_{\lambda_{1}}(x, T x)+k w_{k \lambda}(T x, T y)+\frac{\lambda_{2}}{\lambda} w_{\lambda_{2}}(y, T y)
$$

since $\lambda_{1}+\lambda_{2}=(1-k) \lambda$ and $w_{k \lambda}(T x, T y) \leq w_{\lambda}(x, y)$.

Therefore,

$$
w_{\lambda}(x, y) \leq \frac{\lambda_{1}}{\lambda} w_{\lambda_{1}}(x, T x)+k w_{\lambda}(x, y)+\frac{\lambda_{2}}{\lambda} w_{\lambda_{2}}(y, T y) .
$$

Hence

$$
w_{\lambda}(x, y)=\frac{\lambda_{1} w_{\lambda_{1}}(x, T x)+\lambda_{2} w_{\lambda_{2}}(y, T y)}{\lambda(1-k)}
$$

We now give the first fixed point theorem on modular metric spaces by Chistyakov, but our proof uses the Fundamental Modular Contraction Inequality given in the preceding proposition.

Theorem 4.2 ([3]). Let $w$ be a strict convex modular on $X$ such that the modular space $X_{w}^{*}$ is $w$-complete and let $T: X_{w}^{*} \rightarrow X_{w}^{*}$ be a w-contractive map such that for each $\lambda>0$ there exists an $x=x(\lambda) \in X_{w}^{*}$ such that $w_{\lambda}(x, T x)<\infty$. Then $T$ has a fixed point $x_{*}$ in $X_{w}^{*}$. If the modular $w$ assumes only finite values on $X_{w}^{*}$, then the condition $w_{\lambda}(x, T x)<\infty$ is redundant, the fixed point $x_{*}$ of $T$ is unique and for each $x_{0} \in X_{w}^{*}$ the sequence of iterates $T^{n} x_{0}$ is modular convergent to $x_{*}$.

Proof. If $x, y$ are both fixed point then $w_{\lambda}(x, y)=0$ so $x=y$. Hence a contraction mapping can have at most one fixed point. Its remains to show that for any $x_{0}$ in $X$ the sequence $T^{n}\left(x_{0}\right)$ is Cauchy. Taking $x=T\left(x_{0}\right)$ and $y=T^{n}\left(x_{0}\right)$ and using the Main Contraction Modular Inequality, we get

$$
\begin{aligned}
w_{\lambda}\left(T^{n}\left(x_{0}\right), T^{m}\left(x_{0}\right)\right) \leq & \\
& \frac{\lambda_{1} w_{\lambda_{1}}\left(T\left(T^{n}\left(x_{0}\right), T^{n}\left(x_{0}\right)\right)+\lambda_{2} w_{\lambda_{2}}\left(T\left(T^{m}\left(x_{0}\right), T^{m}\left(x_{0}\right)\right)\right.\right.}{1-k} \\
= & \frac{\lambda_{1} w_{\lambda_{1}}\left(T^{n}\left(T\left(x_{0}\right), T^{n}\left(x_{0}\right)\right)+\lambda_{2} w_{\lambda_{2}}\left(T^{m}\left(T\left(x_{0}\right), T^{m}\left(x_{0}\right)\right)\right.\right.}{1-k} \\
& \leq \frac{\lambda_{1} w_{k^{-n} \lambda_{1}}\left(T\left(x_{0}, x_{0}\right)\right)+\lambda_{2} w_{k^{-m} \lambda_{2}} T\left(x_{0}, x_{0}\right)}{1-k} .
\end{aligned}
$$


Since $k^{-n} \lambda_{1} \rightarrow \infty$ as $n \rightarrow \infty$ then $k^{-n} \lambda_{1}>\lambda$ if $n$ big enough. Similar $k^{-m} \lambda_{2}>\lambda$ if $n$ big enough. Since the function $\lambda \mapsto w_{\lambda}(x, y)$ is non-increasing, and $0<k<1$ we have

$$
w_{k^{-m} \lambda_{2}}\left(T x_{0}, x_{0}\right), w_{k^{-n} \lambda 1}\left(T x_{0}, x_{0}\right) \leq w_{\lambda}\left(T x_{0}, x_{0}\right)<\infty .
$$

Hence

$$
w_{\lambda}\left(T^{n}\left(x_{0}\right), T^{m}\left(x_{0}\right)\right) \rightarrow 0 .
$$

Therefore $T^{n}\left(x_{0}\right)$ is Cauchy.

Chistyakov's second fixed point theorem drops the convexity assumption on the modular, replacing it with the requirement that the mapping be strongly contractive. We start with another variation of Palais's inequality for these mappings.

Proposition 4.3 (Fundamental Strong Modular Contraction Inequality). Let $w$ be a modular on $X$ and let $T: X_{w}^{*} \rightarrow X_{w}^{*}$ be strongly modular contractive mapping, with $w_{k \lambda}(T x, T y) \leq k w_{\lambda}(x, y)$ for $0<\lambda \leq \lambda_{0}$. Let $\lambda_{1}, \lambda_{2} \geq 0, \lambda_{1}+\lambda_{2}=(1-k) \lambda, 0<\lambda<\lambda_{0}$. Then

for all $x, y \in X_{w}^{*}$.

$$
w_{\lambda}(x, y) \leq \frac{w_{\lambda_{1}}(x, T x)+w_{\lambda_{2}}(y, T y)}{1-k}
$$

Proof. By property (3) in the definition of a modular, we get

$$
w_{\lambda_{1}+k \lambda+\lambda_{2}}(x, y) \leq w_{\lambda_{1}}(x, T x)+w_{k \lambda}(T x, T y)+w_{\lambda_{2}}(y, T y)
$$

Using $\lambda_{1}+\lambda_{2}=(1-k) \lambda$ with the strong contractive property of $T$,

$$
w_{\lambda}(x, y) \leq w_{\lambda_{1}}(x, T x)+k w_{\lambda}(x, y)+w_{\lambda_{2}}(y, T y)
$$

and so

for all $x, y \in X_{w}^{*}$.

$$
w_{\lambda}(x, y) \leq \frac{w_{\lambda_{1}}(x, T x)+w_{\lambda_{2}}(y, T y)}{1-k}
$$

We the Fundamental Strong Contraction Modular Inequality (3) to prove the second fixed point theorem.

Theorem 4.4 ([3]). Let $w$ be a strict modular on $X$ such that $X_{w}$ is $w$-complete, let $T: X_{w}^{*} \rightarrow X_{w}^{*}$ be a strongly $w$-contractive map such that for each $\lambda>0$ there exists an $x=x(\lambda) \in X_{w}^{*}$ such that $w_{\lambda}(x, T x)<\infty$ holds. Then $T$ has a fixed point $x_{*}$ in $X_{w}^{*}$. If, in addition, $w$ is finite valued on $X_{w}^{*}$, then the condition $w_{\lambda}(x, T x)<\infty$ is redundant, the fixed point $x_{*}$ of $T$ is unique and for each $x_{0} \in X_{w}^{*}$ the sequence of iterates $T^{n} x_{0}$ is modular convergent to $x_{*}$ [3]. 
Proof. If $x, y$ are both fixed points then $w_{\lambda}(x, y)=0$ so $x=y$. Hence a contraction mapping can have at most one fixed point. Its remains to show that for any $x_{0}$ in $X$ the sequence $T^{n}\left(x_{0}\right)$ is Cauchy. Taking $x=T\left(x_{0}\right)$ and $y=T^{n}\left(x_{0}\right)$,

$$
\begin{aligned}
w_{\lambda}\left(T^{n}\left(x_{0}\right), T^{m}\left(x_{0}\right)\right) \leq \\
\\
\quad \frac{w_{\lambda_{1}}\left(T\left(T^{n}\left(x_{0}\right), T^{n}\left(x_{0}\right)\right)+w_{\lambda_{2}}\left(T\left(T^{m}\left(x_{0}\right), T^{m}\left(x_{0}\right)\right)\right.\right.}{1-k} \\
=\frac{w_{\lambda_{1}}\left(T^{n}\left(T\left(x_{0}\right), T^{n}\left(x_{0}\right)\right)+w_{\lambda_{2}}\left(T^{m}\left(T\left(x_{0}\right), T^{m}\left(x_{0}\right)\right)\right.\right.}{1-k} \\
\quad \leq \frac{k^{n} w_{k^{-n} \lambda 1}\left(T\left(x_{0}, x_{0}\right)\right)+k^{m} w_{k^{-m} \lambda_{2}}\left(T\left(x_{0}, x_{0}\right)\right)}{1-k} .
\end{aligned}
$$

If $k^{-n} \lambda_{1} \rightarrow \infty$ then $k^{-n} \lambda_{1}>\lambda$ if $n$ is big enough. Similarly, $k^{-m} \lambda_{2}>$ $\lambda$ if $n$ also is big enough.

Since the function $\lambda \mapsto w_{\lambda}(x, y)$ is non-increasing, and $0<k<1$ we have

$$
w_{k^{-m} \lambda_{2}}\left(T x_{0}, x_{0}\right), w_{k^{-n} \lambda 1}\left(T x_{0}, x_{0}\right) \leq w_{\lambda}\left(T x_{0}, x_{0}\right)<\infty .
$$

Hence $w_{\lambda}\left(T^{n}\left(x_{0}\right), T^{m}\left(x_{0}\right)\right) \rightarrow 0$. Therefore $T^{n}\left(x_{0}\right)$ is Cauchy.

\section{Applications}

Electrorheological fluids are liquids that rapidly solidify in the presence of an electric field. They are often studied using Sobolev spaces with a variable exponent [8]. It has been suggested that modular metric spaces may be useful in modelling them [1].

Recent work indicates that modular metric space fixed point results are well adapted to certain types of differential equations [4]. Finally, we refer to [5] for a detailed study of nonlinear superposition operators on modular metric spaces of functions.

\section{Acknowledgement}

This article is taken from the first author's MSc thesis, which was written under the supervision of the second author. The first author is grateful to Dr Ryan and to the staff of the School of Mathematics at NUI Galway for all the support and encouragement they gave her. The work of the first author was supported by a grant from the Ministry of Education of Libya, to whom she expresses her gratitude. 


\section{REFERENCES}

[1] Afrah A. N. Abdou and Mohamed A. Khamsi, Fixed point results of pointwise contractions in modular metric spaces, Fixed Point Theory Appl. (2013), 2013:163, 11. MR 3081728

[2] V. V. Chistyakov, Metric modulars and their application, Dokl. Akad. Nauk 406 (2006), no. 2, 165-168. MR 2258511

[3] V. V. Chistyakov, A fixed point theorem for contractions in modular metric spaces, ArXiv e-prints (2011).

[4] Vyacheslav V. Chistyakov, Modular metric spaces. I. Basic concepts, Nonlinear Anal. 72 (2010), no. 1, 1-14. MR 2574913

[5] - Modular metric spaces. II. Application to superposition operators, Nonlinear Anal. 72 (2010), no. 1, 15-30. MR 2574914

[6] Mohamed A. Khamsi, A convexity property in modular function spaces, Math. Japon. 44 (1996), no. 2, 269-279. MR 1416264

[7] Wojciech M. Kozlowski, Modular function spaces, Monographs and Textbooks in Pure and Applied Mathematics, vol. 122, Marcel Dekker, Inc., New York, 1988. MR 1474499

[8] Mihai Mihăilescu and Vicenţiu Rădulescu, On a nonhomogeneous quasilinear eigenvalue problem in Sobolev spaces with variable exponent, Proc. Amer. Math. Soc. 135 (2007), no. 9, 2929-2937. MR 2317971

[9] J. Musielak and W. Orlicz, On modular spaces, Studia Math. 18 (1959), 49-65. MR 0101487

[10] Hidegorô Nakano, Modulared Semi-Ordered Linear Spaces, Maruzen Co., Ltd., Tokyo, 1950. MR 0038565

[11] Richard S. Palais, A simple proof of the Banach contraction principle, J. Fixed Point Theory Appl. 2 (2007), no. 2, 221-223. MR 2372985

[12] M. M. Rao and Z. D. Ren, Applications of Orlicz spaces, Monographs and Textbooks in Pure and Applied Mathematics, vol. 250, Marcel Dekker, Inc., New York, 2002. MR 1890178

Hana Abobaker was a student in the MSc in Mathematics at National University of Ireland Galway in 2015-16, supported by a grant from the Mininstry of Education of Libya. She is currently working in Omar Al-Mukhtar University, Al Bayda, Libya.

Raymond A. Ryan is a long standing member of the School of Mathematics, Statistics \& Applied Mathematics at NUI Galway. He is the author of the book Introduction to Tensor Products of Banach Spaces, published by Springer.

(Hana Abobaker) Omar Al-Mukhtar University, Al Bayda, Libya

(Raymond A. Ryan) School of Mathematics, Statistics \& Applied Mathematics, NUI Galway, Ireland.

E-mail address, Hana Abobaker: libya.first1990@gmail.com

E-mail address, Raymond A. Ryan: ray.ryan@nuigalway.ie 